\title{
Vaccination in healthcare workers: risk assessment, planning, strategy of intervention and legal implications
}

\author{
Venerando Rapisarda ${ }^{1}$, Caterina Ledda*,1(i) \& Helena C Maltezou ${ }^{2}$ \\ ${ }^{1}$ Occupational Medicine, Department of Clinical \& Experimental Medicine, University of Catania, Italy \\ ${ }^{2}$ Department for Interventions in Healthcare Facilities, Hellenic Centre for Disease Control \& Prevention, Athens, Greece \\ *Author for correspondence: cledda@unict.it
}

First draft submitted: 21 August 2018; Accepted for publication: 22 March 2019; Published online: 11 June 2019

Keywords: coverage $\bullet$ healthcare workers $\bullet$ occupational health $\bullet$ policies $\bullet$ training $\bullet$ vaccination

It was our pleasure to chair the Workshop 'Vaccination in healthcare workers: risk assessment, planning, strategy of intervention and legal implications', held in February 2018 in Catania, Italy. The meeting brought together an interdisciplinary group of specialists for an intensive program on vaccination in healthcare workers (HCWs). The topic of this summit reflected the need to stress the importance of vaccinations among HCWs in a particularly difficult period of re-emergence of measles and other vaccine-preventable diseases in Europe and elsewhere, because, transversely, vaccination coverage is decreasing among the general population in the light of the rising antivaccination beliefs and vaccination hesitancy. HCWs serve as a paradigm for their patients and at the same time have the ethical duty to protect them. Their attitudes toward vaccinations are crucial to promoting their uptake, and the successful implementation of routine vaccination programs [1].

The workshop aimed to promote continuous learning and exchange of knowledge, experiences and ideas to increase vaccination in HCWs, as well as promote safety within healthcare facilities. In addition to insights and knowledge to be gained from field experiences, the gaps were clarified and strategies were proposed to raise the immunological protection of vaccine-preventable diseases among HCWs.

\section{Vaccination in HCWs: risk assessment}

Every day, 59 million HCWs are potentially exposed to multiple occupational biological hazards, working with infectious patients, and contaminated fluids and materials [2]. Vaccines are among the most successful and cost effective public health tools and greatly contributed to eliminating or controlling several serious vaccine-preventable diseases in the past century. Not only do vaccines prevent the vaccinated individual from developing a potentially serious illness, but they also help protect entire communities by reducing the spread of infectious agents (herd protection) [3].

It is well known that susceptible HCWs, because of their occupational exposure, have a higher risk of both acquiring and spreading serious vaccine-preventable diseases to vulnerable patients and colleagues, than the general population. Furthermore, the European Center for Disease Prevention and Control states that HCWs are often referred to as the most trusted source of vaccine-related information for their patients and colleagues; it is therefore vital to improve their knowledge and confidence in vaccination programs and vaccines in particular, and engage them in activities targeting the emerging phenomenon of so-called 'vaccine hesitancy' [4]. Vaccine-hesitant HCWs can have a powerful influence on vaccination decisions, as they might recommend vaccines less frequently to their patients, and/or otherwise undermine their confidence, so contributing to vaccine hesitancy among the general population [4]. The role of HCWs can be extremely crucial for the introduction of new vaccines in national vaccination programs (e.g., vaccination against HPV recommended for adolescent girls) and new target groups of patients (e.g., influenza and pertussis vaccination for pregnant women).

HCWs must also be aware that the balance of benefits and risks is dynamic, and may change over time as new data emerge. The benefit-risk balance weighs the benefits of immunization for society (such as the prevention of

Future Medicine 
epidemics, reductions in costs associated with treatment and improved productivity) and those for the individual (prevention of disease and its potential sequelae), against the risks to the individual who might develop an adverse vaccine reaction [5]. To facilitate this assessment, extensive efforts are made to evaluate and ensure a vaccine's safety from early development through its entire duration of use. At licensure, surveillance activities are put in place to continue monitoring safety and disease epidemiology, and to supply reliable and up-to-date information to maintain public confidence in immunization programs [5]. Among all stakeholders, HCWs play a critical role that includes identifying adverse events following immunization, collecting all available clinical information related to the adverse events following immunization and reporting the event, including any evaluation of risk factors that may have contributed to the event [5].

\section{Vaccination in HCWs: planning}

The EU and national occupational regulations as well as the joint WHO and International Labour Organization guidelines indicate that prophylaxis is the responsibility of the employer [2].

The Italian National Vaccination Plan, and also vaccination programs from many countries, strongly recommend that HCWs be offered hepatitis B and influenza vaccines and that susceptible workers should also be offered measles, mumps, rubella and varicella vaccines [6]. The inclusion of specific vaccinations in the National Vaccination Programs for HCWs should also consider the current epidemics and gaps in immunity among them, as well as the increased movement of populations across borders [1]. Moreover, TB prevention procedures are a consolidated practice for occupational health physicians. Hospital health directors should be made aware of the importance of vaccinating HCWs as a milestone in hospital risk management. More adequate training, including specific courses on vaccinations, is required for occupational health physicians [3,7-9].

Hospital health directors should have vaccination registries for HCWs, including details of HCWs' past history of vaccine preventable diseases; vaccinations; antibody and test results (for example, Mantoux status); record of vaccines consented/refused or nonresponse to vaccination; and batch number and brand name of vaccine. This should be updated when new events (vaccination, test, disease) occur and should be managed by a designated staff member [5].

However, despite the evidence of the protective effects of vaccination, coverage in HCWs remains suboptimal. In addition to morbidity and mortality for them and their vulnerable patients (e.g., newborns, the elderly, pregnant women, etc.), healthcare-associated outbreaks can negatively impact on essential health services through staff absenteeism.

\section{Vaccination in HCWs: strategy of intervention}

The WHO has indicated that training is an essential component of all expanded programs on immunization activities [2]. Successful interventions may be useful to change the attitudes and practices of HCWs toward vaccinepreventable diseases and vaccinations for them and the general population, and increase their immunization coverage rates. The aim of this program was to train instructors who, after completing their educational term, would be able to pass basic knowledge on immunization on to primary HCWs. The second step of the program was to disseminate this practice to all HCWs [9-11].

Training may help HCWs update their knowledge about immunization and vaccination. Additional strategies include electronic communication, bespoke stickers to be placed on the staff identification badges of vaccinated HCWs, and posters and screensavers for network computers displayed in hospitals, for example [12]. Messages to encourage vaccination that focus on protection against vaccine-preventable diseases make it easier to help HCWs approach vaccination $[13,14]$.

\section{Vaccination in HCWs: legal implications}

Issues with vaccinations can simply be caused by the lack of proper information, and the dissemination of misconceptions about vaccine efficacy and safety. However, the promotion of vaccinations for HCWs and the general population also involves several ethical and legal issues; first of all the potential disagreement between individual and collective rights $[1,15,16]$.

Currently in Italy, but also in other European countries, the legal nature of vaccinations for HCWs is being strongly debated because of the serious immunity gaps against several vaccine-preventable diseases of major importance for healthcare facilities, which is at the center of the bioethical debate. However, this exacerbates the conflict between the good of the individual versus the good of the community $[17,18]$. 
Italian law recently provided an obligation for vaccination but it has not entirely clarified logistical aspects for the healthcare setting. The issue of mandatory vaccinations for HCWs should also be addressed in other countries, in light of recent measles epidemics and increasing vaccination hesitancy in Europe.

\section{Conclusion}

In this special issue, hot topics will be addressed and it is hoped that they will be helpful to the whole healthcare community and patients. We thank our colleagues for their valuable contribution.

\section{Financial \& competing interests disclosure}

The authors have no relevant affiliations or financial involvement with any organization or entity with a financial interest in or financial conflict with the subject matter or materials discussed in the manuscript. This includes employment, consultancies, honoraria, stock ownership or options, expert testimony, grants or patents received or pending, or royalties.

No writing assistance was utilized in the production of this manuscript.

\section{References}

1 Maltezou HC, Poland GA. Vaccination policies for healthcare workers in Europe. Vaccine 32(38), 4876-4880 (2014).

2 World Health Organization. Occupational health. Health Workers. www.who.int/occupational_health/topics/hcworkers/en/

3 Di Pasquale A, Bonanni P, Garçon N et al. Vaccine safety evaluation: practical aspects in assessing benefits and risks. Vaccine 34(52), 6672-6680 (2016).

4 Larson HJ, Jarrett C, Eckersberger E et al. Understanding vaccine hesitancy around vaccines and vaccination from a global perspective: a systematic review of published literature, 2007-2012. Vaccine 32(19), 2150-2159 (2014).

5 Di Pasquale A, Bonanni P, Garçon N et al. Vaccine safety evaluation: practical aspects in assessing benefits and risks. Vaccine 34(52), 6672-6680 (2016).

6 Italian Ministry of Health. National vaccination prevention plan 2017-2019. www.salute.gov.it/portale/documentazione/p6_2_2_1.jsp?lingua=italiano\&id=2571

7 Prato R, Tafuri S, Fortunato F, Martinelli D. Vaccination in healthcare workers: an Italian perspective. Expert Rev. Vaccines 9(3), 277-283 (2010).

8 Loulergue P, Moulin F, Vidal-Trecan G et al. Knowledge, attitudes and vaccination coverage of healthcare workers regarding occupational vaccinations. Vaccine 27(31), 4240-4243 (2009).

9 Marotta C, Raia DD, Ventura G et al. Improvement in vaccination knowledge among health students following an integrated extra curricular intervention, an explorative study in the University of Palermo. J. Prev. Med. Hyg. 58(2), E93-E98 (2017).

10 Ramaci T, Pellerone M, Ledda C et al. Health promotion, psychological distress, and disease prevention in the workplace: a cross-sectional study of Italian adults. Risk Manag. Healthcare Pol. 10, 167-175 (2017).

11 Uskun E, Uskun SB, Uysalgenc M, Yagiz M. Effectiveness of a training intervention on immunization to increase knowledge of primary healthcare workers and vaccination coverage rates. Public Health 122(9), 949-958 (2008).

12 Heinrich-Morrison K, McLellan S, McGinnes U et al. An effective strategy for influenza vaccination of healthcare workers in Australia: experience at a large health service without a mandatory policy. BMC Infect. Dis. 15(1), 42 (2015).

13 Elder RW. Combination of mass media health campaigns and health-related product distribution is recommended to improve healthy behaviors. Am. J. Prev. Med. 47(3), 372-374 (2014).

14 Chean R, Ferguson JK, Stuart RL. Mandatory seasonal influenza vaccination of health care workers: a way forward to improving influenza vaccination rates. Healthcare Infect. 19(2), 42-44 (2014).

15 Ledda C, Cicciù F, Puglisi B et al. Attitude of health care workers (HCWs) toward patients affected by HIV/AIDS and drug users: a cross-sectional study. Int. J. Environ. Res. Public. Health 14(3), pii:E284 (2017).

16 Maltezou HC, Poland GA. Immunization of healthcare providers: a critical step toward patient safety. Vaccine 32(38), 4813 (2014).

17 Hatzianastasiou S, Pavli A, Maltezou HC. Legal aspects of public health: how law frames communicable disease control in Greece. $J$. Public Healthcare Pol. 32(4), 445-457 (2011).

18 Maltezou HC, Tsakris A. Vaccination of health-care workers against influenza: our obligation to protect patients. Influ. Other Respir. Viruses 5(6), 382-388 (2011). 
